\title{
Elliptic flow of gluon matter in ultrarelativistic heavy-ion collisions
}

\author{
Zhe $\mathrm{Xu}^{*}$ and Carsten Greiner \\ Institut für Theoretische Physik, Goethe-Universität Frankfurt, \\ Max-von-Laue-Strasse 1, D-60438 Frankfurt am Main, Germany
}

(Dated: November 6, 2018)

\begin{abstract}
Employing a perturbative QCD based parton cascade we calculate the elliptic flow $v_{2}$ and its transverse momentum dependence $v_{2}\left(p_{T}\right)$ for the gluon matter created in $\mathrm{Au}+\mathrm{Au}$ collisions at $\sqrt{s_{N N}}=200 \mathrm{GeV}$. To make comparisons with the experimental data at the BNL Relativistic Heavy Ion Collider (RHIC), parton-hadron duality is assumed. We find that whereas the integrated $v_{2}$ matches the experimental data, the gluon (or pion) $v_{2}\left(p_{T}\right)$ is about $20-50 \%$ smaller than the experimental data. Hadronization via gluon fragmentation and quark recombination seems to be the key to explaining the necessary jump of $v_{2}\left(p_{T}\right)$ from the partonic to the hadronic phase. We also show that the elliptic flow values moderately depend on the chosen freezeout condition, which will thus constrain the shear viscosity to the entropy density ratio of the quark gluon plasma created at RHIC.
\end{abstract}

PACS numbers: 25.75.-q, 25.75.Ld, 12.38.Mh, 24.10.Lx

\footnotetext{
*xu@th.physik.uni-frankfurt.de
} 


\section{INTRODUCTION}

Elliptic flow [1, 2] is the key observable characterizing the collectivity [3] of the quark gluon plasma (QGP) that is potentially produced in ultrarelativistic heavy-ion collisions at the BNL Relativistic Heavy Ion Collider (RHIC) or/and at the CERN Large Hadron Collider (LHC). A fast transformation from the initial spatial anisotropy to the momentum anisotropy will indicate sufficiently strong interactions among quarks and gluons, which also provides the reason for a fast thermalization to a QGP with a small shear viscosity. The good agreements of the elliptic flow $v_{2}$ data at RHIC [4, 5] with the results from calculations employing ideal hydrodynamics [6] suggest that the shear viscosity of the QGP created at RHIC is small [7]. To find its lower and upper bound is an important but difficult task, because the elliptic flow is built up not only during the evolution of the QGP but also initially during the thermalization and finally, though possibly marginal, during the hadronic cascade before particles decouple kinetically. Moreover, the lack of understanding of the hadronization leads to an uncertainty in converting the partonic elliptic flow to the hadronic one that is measured. All these contributions to the final hadronic elliptic flow can, in principle, be investigated in a transport calculation including a partonic and a hadronic cascade and a hadronization mechanism [8]. Such studies will help to determine the true shear viscosity in the QGP phase.

Recently, employing the perturbative QCD (pQCD) based parton cascade Boltzmann Approach of MultiParton Scatterings (BAMPS) [9, 10, 11], we have calculated the elliptic flow $v_{2}$ of the gluon matter produced in $\mathrm{Au}+\mathrm{Au}$ collisions at the RHIC energy [12]. To compare our results with the experimental data the gluonic $v_{2}$ was converted to the hadronic one using the parton-hadron duality picture at a freezeout energy density of $e_{c}=1 \mathrm{GeV} \mathrm{fm}^{-3}$. Hence $v_{2}^{\text {pion }}\left(p_{T}\right)=v_{2}^{\text {gluon }}\left(p_{T}\right)$ at transverse momenta $p_{T}$. Even though the hadronization was described in such a simple manner, it was found that when employing a QCD coupling constant of $\alpha_{s}=0.6$, the calculated $v_{2}$ matches the experimental data [13, 14]. With $\alpha_{s}=0.3$ the results are about $20 \%$ smaller than the data. Hence, pQCD interactions can explain

the large $v_{2}$ buildup at RHIC. In addition, in Refs. [12] and [15] the shear viscosity to the entropy density ratio $\eta / s$ from the transport calculations was extracted and it was found that $\eta / s$ is between 0.15 for $\alpha_{s}=0.3$ and 0.08 for $\alpha_{s}=0.6$. These findings are similar to those obtained from viscous hydrodynamical calculations [16]. 
This article provides details on our recent results on the elliptic flow from parton cascade calculations. In Sec. [I] setups for numerical calculations are given. Section [II shows the results for the transverse momentum dependence of the elliptic flow, $v_{2}\left(p_{T}\right)$, the transverse momentum spectra, the mean transverse momentum, and the final transverse energy per rapidity at midrapidity. The results from the BAMPS calculations are compared with the experimental data at RHIC assuming parton-hadron duality. Further possible improvements as well as a discussion about possible hadronization scenarios via gluon fragmentation and quark recombination are given. In Sec. IV the dependence of the elliptic flow on the chosen freezeout energy density is presented, which indicates the uncertainty of the $\eta / s$ ratio extracted from the BAMPS calculations. Finally we summarize in Sec. V. In Appendix $\mathrm{A}$, the calculation of the number of participating nucleons and the determination of centrality classes are given.

\section{BAMPS SETUPS}

BAMPS solves the Boltzmann equation for on-shell gluons with pQCD interactions, which include elastic scatterings and bremsstrahlung and its backreaction. The cross section of pQCD elastic scatterings is given by [9, 17, 18]

$$
\frac{d \sigma^{g g \rightarrow g g}}{d \mathbf{q}_{\perp}^{2}}=\frac{9 \pi \alpha_{s}^{2}}{\left(\mathbf{q}_{\perp}^{2}+m_{D}^{2}\right)^{2}},
$$

where $\mathbf{q}_{\perp}$ denotes the perpendicular component of the momentum transfer in the center-of-

mass frame of the elastic collision. The interactions are screened by a Debye mass [9, 17, 18],

$$
m_{D}^{2}(\mathbf{x}, t)=\pi d_{G} \alpha_{s} N_{c} \int \frac{d^{3} p}{(2 \pi)^{3}} \frac{f(\mathbf{x}, t, \mathbf{p})}{p},
$$

where $d_{G}=16$ is the gluon degeneracy factor for $N_{c}=3 . m_{D}$ is calculated locally using the gluon density function $f(\mathbf{x}, t, \mathbf{p})$ obtained from the BAMPS simulation. The integrated cross section is

$$
\sigma^{g g \rightarrow g g}=\frac{1}{2} \int_{0}^{s / 4} d \mathbf{q}_{\perp}^{2} \frac{d \sigma^{g g \rightarrow g g}}{d \mathbf{q}_{\perp}^{2}}=\frac{9}{2} \frac{\pi \alpha_{s}^{2}}{m_{D}^{2}\left(1+4 m_{D}^{2} / s\right)},
$$

and $s$ is the invariant mass of the collision. The factor $1 / 2$ before the integral indicates that the outgoing particles in the collision are identical.

The effective matrix element of pQCD inspired bremsstrahlung $g g \leftrightarrow g g g$ is taken in a 
Gunion-Bertsch form [17, 18, 19],

$$
\left|\mathcal{M}_{g g \rightarrow g g g}\right|^{2}=\frac{9 g^{4}}{2} \frac{s^{2}}{\left(\mathbf{q}_{\perp}^{2}+m_{D}^{2}\right)^{2}} \frac{12 g^{2} \mathbf{q}_{\perp}^{2}}{\mathbf{k}_{\perp}^{2}\left[\left(\mathbf{k}_{\perp}-\mathbf{q}_{\perp}\right)^{2}+m_{D}^{2}\right]} \Theta\left(k_{\perp} \Lambda_{g}-\cosh y\right),
$$

where $g^{2}=4 \pi \alpha_{s} . \mathbf{k}_{\perp}$ and $y$ denote the perpendicular component of the radiated gluon momentum and its rapidity in the center-of-mass frame of the collision, respectively. $k_{\perp}=\left|\mathbf{k}_{\perp}\right|$, and $\Lambda_{g}$ is the gluon mean free path, which is calculated self-consistently [9]. The suppression of the bremsstrahlung due to the Landau-Pomeranchuk-Migdal effect is effectively taken into account within the Bethe-Heitler regime using the step function in Eq. (4). Gluon radiations and absorptions are only allowed if the formation time of the process, typically $\tau=\cosh y / k_{\perp}$, is shorter than the mean free path of the radiated or absorbed gluon. Both the Debye screening mass and the gluon mean free path act as an infrared regulator.

The total cross section for bremsstrahlung is obtained by the integral

$$
\sigma_{g g \rightarrow g g g}=\frac{1}{2 s} \frac{1}{3 !} \int d \Gamma_{1}^{\prime} d \Gamma_{2}^{\prime} d \Gamma_{3}^{\prime}\left|\mathcal{M}_{g g \rightarrow g g g}\right|^{2}(2 \pi)^{4} \delta^{(4)}\left(p_{1}+p_{2}-p_{1}^{\prime}-p_{2}^{\prime}-p_{3}^{\prime}\right)
$$

where $d \Gamma_{i}^{\prime} \equiv d^{3} p_{i}^{\prime} /(2 \pi)^{3} /\left(2 E_{i}^{\prime}\right) . p_{1}$ and $p_{2}$ denote the four-momenta of the incoming gluons, and $p_{i}^{\prime}, i=1,2,3$ denote the four-momenta of the outgoing gluons. For the backreactions we define a "cross section" by [9]

$$
I_{g g g \rightarrow g g}=\frac{1}{2} \int d \Gamma_{1}^{\prime} d \Gamma_{2}^{\prime}\left|\mathcal{M}_{g g g \rightarrow g g}\right|^{2}(2 \pi)^{4} \delta^{(4)}\left(p_{1}+p_{2}+p_{3}-p_{1}^{\prime}-p_{2}^{\prime}\right),
$$

with $\left|\mathcal{M}_{g g g \rightarrow g g}\right|^{2}=\left|\mathcal{M}_{g g \rightarrow g g g}\right|^{2} / d_{G}$.

Interactions are simulated by the stochastic method [9, 20, 21, 22, 23] according to the interaction (i.e., transition) probabilities

$$
\begin{aligned}
P_{g g \rightarrow g g} & =v_{\text {rel }} \frac{\sigma_{g g \rightarrow g g}}{N_{\text {test }}} \frac{\Delta t}{\Delta^{3} x}, \\
P_{g g \rightarrow g g g} & =v_{\text {rel }} \frac{\sigma_{g g \rightarrow g g g}}{N_{\text {test }}} \frac{\Delta t}{\Delta^{3} x}, \\
P_{g g g \rightarrow g g} & =\frac{1}{8 E_{1} E_{2} E_{3}} \frac{I_{g g g \rightarrow g g}}{N_{\text {test }}^{2}} \frac{\Delta t}{\left(\Delta^{3} x\right)^{2}},
\end{aligned}
$$

for $g g \rightarrow g g, g g \rightarrow g g g$, and $g g g \rightarrow g g$ processes, respectively. $v_{\text {rel }}=s /\left(2 E_{1} E_{2}\right)$ is the relative velocity between incoming gluons. $\Delta^{3} x$ denotes the volume of the cell element and $\Delta t$ the time step. The probabilities are calculated for every doublet and triplet in each spatial cell and are compared with random numbers between 0 and 1 to determine whether or not interactions occur. If the random number is smaller than the probability for an interaction, 
the interaction occurs and new momenta of outgoing gluons will be sampled according to Eqs. (11) or (4). Details of the samplings can be found in Ref. [9]. If the random number is larger than the probability for an interaction, no interaction will occur. Particles that do not participate in interactions within $\Delta t$ will propagate as a free streaming. These operations run over all the cells and time steps until the freezeout condition is reached.

To reduce numerical artifacts, the cell length is set to be smaller than the gluon mean free path. For instance, in noncentral $\mathrm{Au}+\mathrm{Au}$ collisions with an impact parameter of $b=8.6 \mathrm{fm}$, the transverse cell length is set to be a constant small value of $\Delta x=\Delta y=0.125 \mathrm{fm}$. The setup of the longitudinal cell length is refreshed before each new time step so that there are almost the same number of particles in each $\Delta z$ bin with $\Delta z=t\left[\tanh \left(\eta_{s 2}\right)-\tanh \left(\eta_{s 1}\right)\right]$, where $t$ is the time in the center-of-mass frame of a $\mathrm{Au}+\mathrm{Au}$ collision and $\eta_{s}=\frac{1}{2} \ln [(t+z) /(t-z)]$ denotes the space time rapidity. It turns out that the longitudinal cells are almost equidistant in $\eta_{s}[10]$, i.e., $\left|\eta_{s 2}-\eta_{s 1}\right| \approx$ const, which implies a nearly Bjorken-type space time evolution of the gluon matter.

The accurate solution of the Boltzmann equation using the stochastic method according to Eqs. (7) - (91) is guaranteed if there are enough particles in each cell, because this condition is assumed when deriving the interaction probabilities Eqs. (7) - (9) [9]. Because the cell volume has to be small, the real gluon number in each cell is too small to fulfill the condition for using the stochastic method. We adopt the test particle method to amplify the particle number with a factor of $N_{\text {test }}$. To leave the physical scale of the gluon mean free path unchanged, the interaction probabilities are accordingly reduced by $N_{\text {test }}$ and $N_{\text {test }}^{2}$, respectively [see Eqs. (7)-(9)]. One "event" means $N_{\text {test }}$ events with parallel propagations. Particles from different events can interact but with the reduced probabilities, so that each particle still behaves as a true physical one. On the other hand, when calculating the real particle number and energy density, the values must be divided by $N_{\text {test }}$. We set $N_{\text {test }}=2400$ for $b=8.6 \mathrm{fm}$, which leads to $\left|\eta_{s 2}-\eta_{s 1}\right| \approx 0.1$ for the longitudinal cell length.

In the present pQCD simulations the interactions of the gluons are stopped when the local energy density drops below $e_{c}$. The value for $e_{c}$ is assumed to be the critical value for the occurrence of hadronization, below which parton dynamics is not valid. Because a realistic hadronization and a hadronic cascade are not yet included in BAMPS, a gluon, which ceases to interact, propagates freely and is regarded as a free pion according to the parton-hadron duality picture ( $\delta$-function fragmentation of the gluon). Thus, $e_{c}$ determines the freezeout 
condition. The implementation of a Cooper-Frye prescription for hadronization [24] and the subsequent UrQMD [25] hadronic cascade after the QGP evolution is in progress.

Because the implementation of Bose enhancement into transport calculations is still technically difficult, gluons are treated as Boltzmann particles for simplicity. If the gluon system is in thermal equilibrium, the energy density is $e=48 T^{4} / \pi^{2}$. In Ref. [12] we have chosen $e_{c}=1 \mathrm{GeV} \mathrm{fm}^{-3}$, which leads to a critical temperature of $T_{c}=200 \mathrm{MeV}$. To see the possible dependence of the elliptic flow on the freezeout density we choose, in this article, an additional moderately lower value of $e_{c}=0.6 \mathrm{GeV} \mathrm{fm}^{-3}$ that gives $T_{c}=175 \mathrm{MeV}$.

The initial gluon distribution for BAMPS calculations is the same as that chosen in

Ref. [12]: an ensemble of minijets [26] with transverse momenta greater than $p_{0}=1.4 \mathrm{GeV}$, produced via semihard nucleon-nucleon collisions. The value of $p_{0}$ is chosen by matching the final transverse energy per rapidity from the parton cascade calculations to the experimental data [12]. We use Glauber geometry with a Woods-Saxon profile and assume independent binary nucleon-nucleon collisions. The inclusion of quarks in BAMPS is straightforward and is in progress. We do not expect large changes in the integrated elliptic flow $v_{2}$, because the quark amount at RHIC is initially about $20 \%$ only. As quarks interact weaker than gluons, we expect that $v_{2}$ will be slightly smaller than that obtained in the present pure gluon matter approach. More discussion about this issue appears at the end of the next section.

\section{ELLIPTIC FLOW AND DISCUSSIONS ON HADRONIZATION}

\section{A. Results}

The BAMPS calculations for $\mathrm{Au}+\mathrm{Au}$ collisions at the RHIC energy $\sqrt{s_{N N}}=200 \mathrm{GeV}$ are carried out for a set of discrete impact parameters from $b=2$ to $11 \mathrm{fm}$ (see Appendix A). The corresponding number of participating nucleons $N_{\text {part }}(b)$ is calculated within the wounded nucleon model [27]. The details are presented in Appendix A. The results from the BAMPS calculations with the given impact parameters are used to obtain the elliptic flow as a function of the transverse momentum $v_{2}\left(p_{T}\right)$ and the transverse momentum spectra in different centrality classes. Calculations with randomly sampled impact parameters are not appropriate for BAMPS, because a single event is already extremely time consuming for 
large test particle numbers $N_{\text {test }}$.

Figure 1 shows the elliptic flow $v_{2}=\left\langle\left(p_{x}^{2}-p_{y}^{2}\right) / p_{T}^{2}\right\rangle$ as a function of the number of participating nucleons, $N_{\text {part }}$. The points are STAR [13] and PHOBOS [14] data for charged

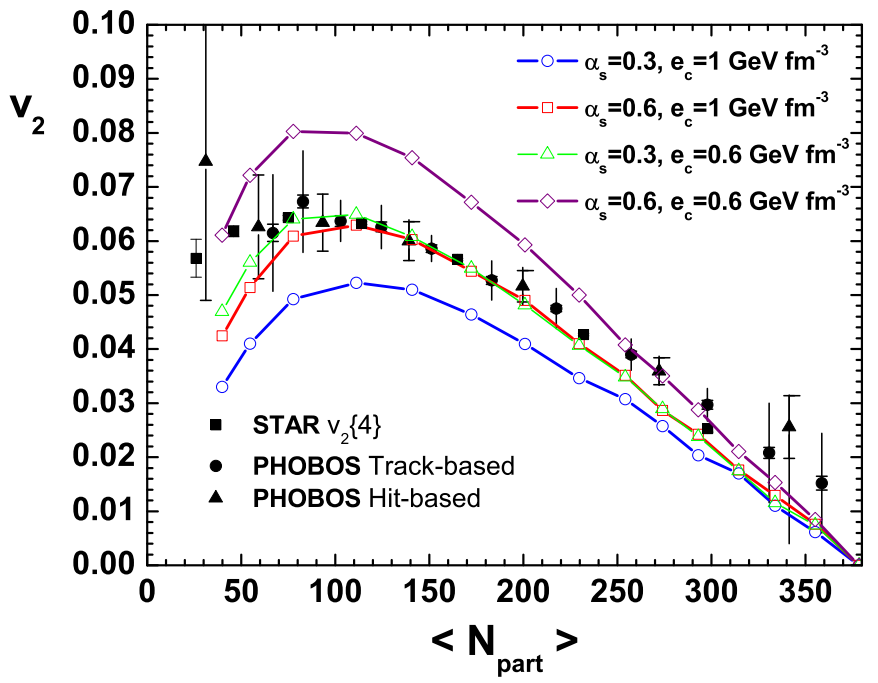

FIG. 1: (Color online) Elliptic flow vs $N_{\text {part }}$ for $\mathrm{Au}+\mathrm{Au}$ collisions at $\sqrt{s_{N N}}=200 \mathrm{GeV}$. The points are STAR [13] and PHOBOS [14] data for charged hadrons within $|\eta|<0.5$ and $|\eta|<1$, respectively, whereas the curves with symbols are results for gluons within $|\eta|<1$, obtained from the BAMPS calculations with $\alpha_{s}=0.3$ and 0.6 and with two freezeout energy densities, $e_{c}=0.6$ and $1 \mathrm{GeV} \mathrm{fm}^{-3}$.

hadrons within the pseudorapidity intervals $|\eta|<0.5$ and $|\eta|<1$, respectively. The symbols, which are connected with colored straight lines, are results for gluons within $|\eta|<1$ ( $\eta$ being identical to the momentum rapidity $y$ for massless gluons) from the BAMPS calculations for two values of the coupling constant $\alpha_{s}$ and for two values of the critical energy density $e_{c}$ for the freezeout. Especially, the blue curve with open circles and the red curve with open squares are the results for $\left(\alpha_{s}=0.3, e_{c}=1 \mathrm{GeV} \mathrm{fm}^{-3}\right)$ and $\left(\alpha_{s}=0.6, e_{c}=1 \mathrm{GeV} \mathrm{fm}^{-3}\right)$, respectively. These are exactly the same as shown in Fig. 2 of Ref. [12]. The new results for $e_{c}=0.6 \mathrm{GeV} \mathrm{fm}^{-3}$ will be discussed in the next section.

Figure 2 shows the elliptic flow as a function of the transverse momentum $v_{2}\left(p_{T}\right)$ for the most central $50 \%$ of $\mathrm{Au}+\mathrm{Au}$ collisions at $\sqrt{s_{N N}}=200 \mathrm{GeV}$. The points are PHOBOS data [14] for charged hadrons within $0<\eta<1.5$, whereas the curves with symbols are results for gluons within $|\eta|<1.5$ from BAMPS calculations. Figure 3 shows $v_{2}\left(p_{T}\right)$ in different 


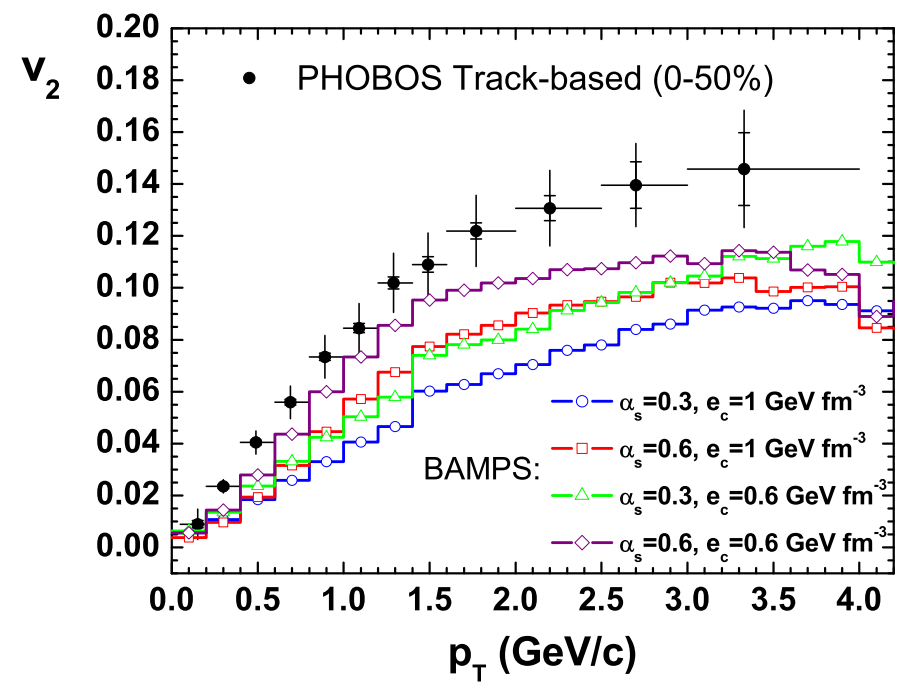

FIG. 2: (Color online) Elliptic flow vs transverse momentum for the most central $50 \%$ of $\mathrm{Au}+\mathrm{Au}$ collisions at $\sqrt{s_{N N}}=200 \mathrm{GeV}$. The points are PHOBOS data [14] for charged hadrons within $0<\eta<1.5$, whereas the curves with symbols are results for gluons within $|\eta|<1.5$, obtained from the BAMPS calculations with $\alpha_{s}=0.3$ and 0.6 and with $e_{c}=0.6$ and $1 \mathrm{GeV} \mathrm{fm}^{-3}$.

centrality classes for particles within $|\eta|<0.5$. The PHENIX [4, 28] and STAR data [13] are depicted for comparisons with the results from the BAMPS calculations. The latter are plotted until $p_{T}=4 \mathrm{GeV}$, because the statistical errors at higher $p_{T}$ are too large.

Although the integrated $v_{2}$ of gluons from the BAMPS calculations with $\alpha_{s}=0.6$ and $e_{c}=1 \mathrm{GeV} \mathrm{fm}^{-3}$ agree perfectly with the experimental data (see the red curve with open squares in Fig. 1), the differential $v_{2}\left(p_{T}\right)$ (the red curve with open squares in Fig. 2 and red curves in Fig. 3) are 20-50\% smaller than the data, especially at intermediate momenta $1.5 \mathrm{GeV}<p_{T}<4 \mathrm{GeV}$. Correspondingly, the $p_{T}$ spectra of gluons, shown in Fig. 4, are flatter than those of hadrons at RHIC. The larger the centrality, the larger is the difference between the data and the results from the calculations.

Discrepancy with the RHIC data is also seen in the mean transverse momentum, $\left\langle p_{T}\right\rangle$, which is shown in the upper panel of Fig. [5 as a function of $N_{\text {part }}$. The points are STAR data [30] within $0<\eta<1$, whereas the curves with symbols are the BAMPS results for gluons within $|\eta|<1$. The mean $p_{T}$ of gluons is larger than that of charged hadrons. On the other hand, the total transverse energy of gluons per rapidity at midrapidity, shown in the lower panel of Fig. 5, agrees with the experimental data including hadronic and electromagnetic 


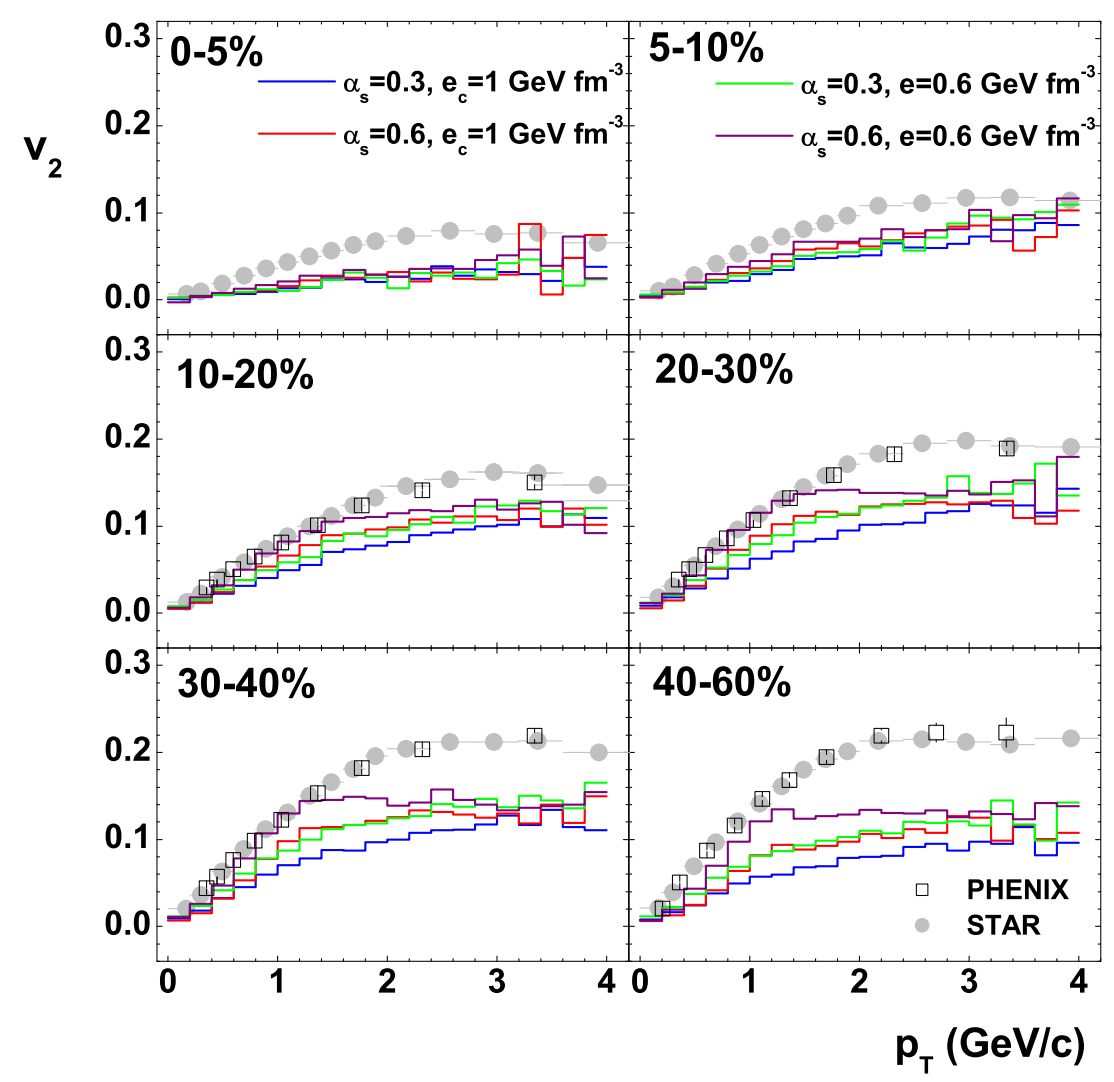

FIG. 3: (Color online) Elliptic flow vs transverse momentum for different centrality bins of $\mathrm{Au}+\mathrm{Au}$ collisions at $\sqrt{s_{N N}}=200 \mathrm{GeV}$. The points are PHENIX [4, 28] and STAR data [13] for charged hadrons within $|\eta|<0.35$ and $|\eta|<0.5$, whereas the curves are results for gluons within $|\eta|<0.5$, obtained from the BAMPS calculations with $\alpha_{s}=0.3$ and 0.6 and with $e_{c}=0.6$ and $1 \mathrm{GeV} \mathrm{fm}^{-3}$. components [30]. We notice that the results of $d E_{T} / d \eta$ with a larger coupling constant of $\alpha_{s}=0.6$ are $7-15 \%$ smaller than those with $\alpha_{s}=0.3$ (also smaller than the data) because of the larger longitudinal work during a less viscous hydrodynamical expansion. However, if quarks are included in BAMPS calculations, the transverse energy will be slightly enlarged.

\section{B. Discussion}

$d E_{T} / d \eta$ agrees rather well with the data but $\left\langle p_{T}\right\rangle$ is larger than the data. This thus indicates that the gluon number per rapidity is smaller than that of the hadron number per rapidity. The parton-hadron duality with one gluon to one pion seems too simple to describe the complex hadronization. To obtain $\left\langle p_{T}\right\rangle$ that agrees with the data, each gluon 


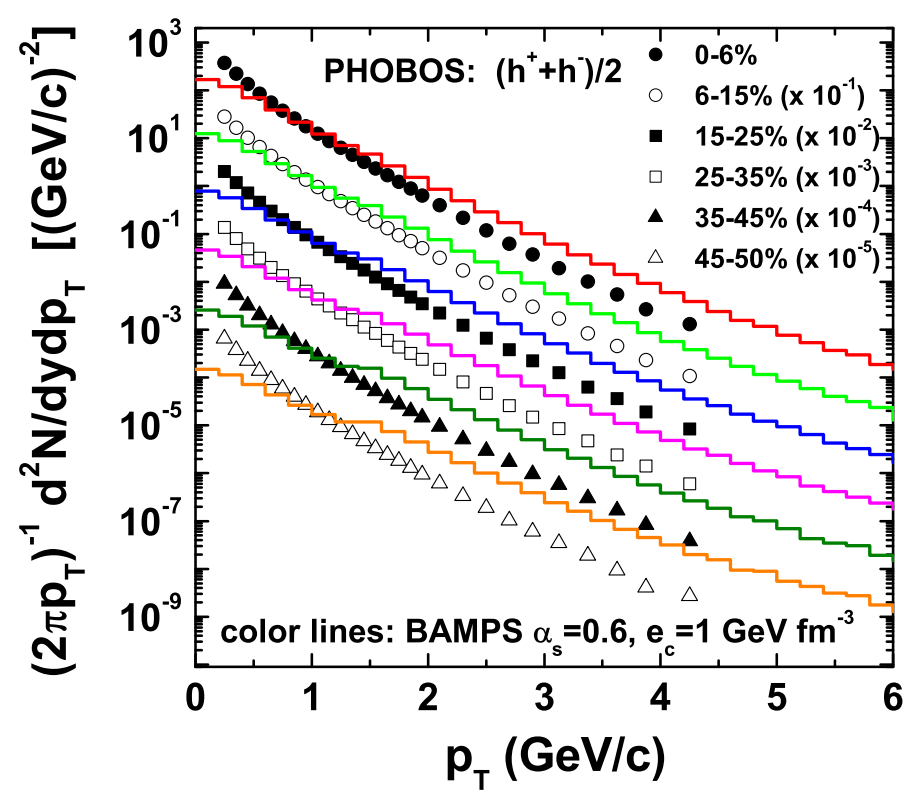

FIG. 4: (Color online) Transverse momentum spectra for different centrality bins of Au+Au collisions at $\sqrt{s_{N N}}=200 \mathrm{GeV}$. The points are PHOBOS data [29] for charged hadrons within the rapidity interval $0.2<y_{\pi^{ \pm}}<1.4$, whereas the curves are results for gluons within $|y|<1.5$, obtained from the BAMPS calculations with $\alpha_{s}=0.6$ and $e_{c}=1 \mathrm{GeV} \mathrm{fm}^{-3}$. To compare with the experimental data, the gluon number is reduced by a factor of 3 .

is expected to "fragment" to 1.5-2 pions on average. The larger the centrality (peripheral collisions), the more fragments are needed, because initial hard gluon jets are less quenched in peripheral collisions because of their small reaction size.

Possible fragmentation of each gluon to more pions suggests $v_{2}^{\text {pion }}\left(p_{T}\right) \approx v_{2}^{\text {gluon }}\left(n p_{T}\right)$ and $d N^{\text {pion }}\left(p_{T}\right) \sim d N^{\text {gluon }}\left(n p_{T}\right)$ with $n=1.5-2$. The obtained $v_{2}^{\text {pion }}\left(p_{T}\right)$ for low $p_{T}<1 \mathrm{GeV}$ would come closer to the experimental data. Because the integrated $v_{2}$ is determined mostly by the lower $p_{T}$ particles, the integrated $v_{2}$ of pions will be nearly the same as that of gluons. On the other hand, the $v_{2}^{\text {pion }}\left(p_{T}\right)$ for $p_{T}>1 \mathrm{GeV}$ would be still $20-50 \%$ smaller than the data. The discrepancy is larger for more peripheral collisions (see Fig. 3). It seems that quark recombination models [31, 32, 33, 34] for hadronization are appropriate for intermediate $p_{T}$ gluons, if one assumes that each gluon must first be converted to a quark-antiquark pair $g \rightarrow q \bar{q}$ via $v_{2}^{\text {quark }}\left(p_{T} / 2\right) \approx v_{2}^{\text {gluon }}\left(p_{T}\right)$. Then the quark recombination models would give $\left.v_{2}^{\text {pion }}\left(p_{T}\right) \approx 2 v_{2}^{\text {quark }}\left(p_{T} / 2\right)\right) \approx 2 v_{2}^{\text {gluon }}\left(p_{T}\right)$.

We also must stress that the results discussed above are obtained from the present calcu- 

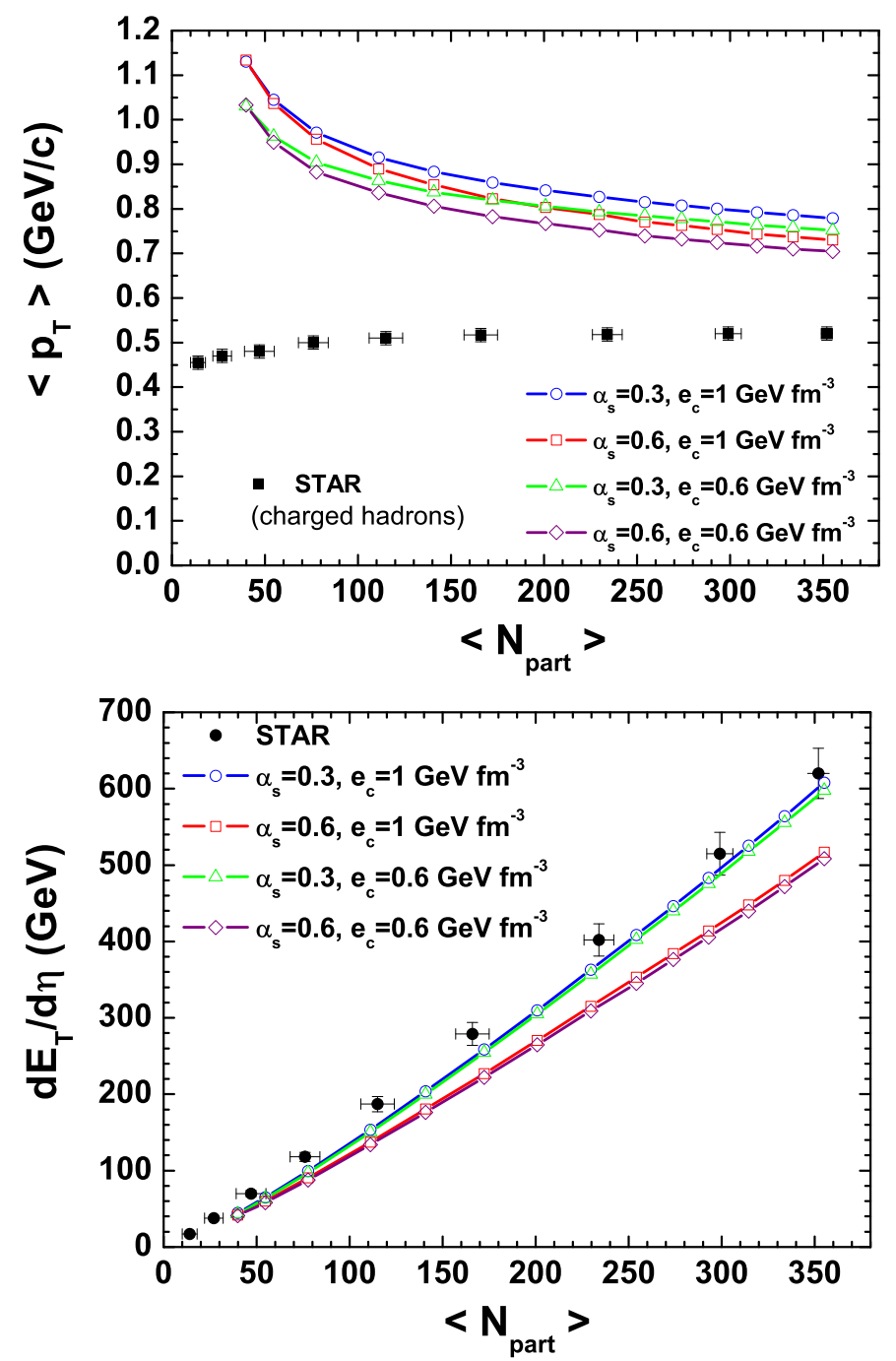

FIG. 5: (Color online) Mean transverse momentum vs $N_{\text {part }}$ (upper panel) and transverse energy per pseudorapidity vs $N_{\text {part }}$ (lower panel) in $\mathrm{Au}+\mathrm{Au}$ collisions at $\sqrt{s_{N N}}=200 \mathrm{GeV}$. The points are STAR data 30] within $0<\eta<1$, whereas the curves with symbols are results for gluons within $|\eta|<1$, obtained from the BAMPS calculations with $\alpha_{s}=0.3$ and 0.6 and with $e_{c}=0.6$ and 1 $\mathrm{GeV} \mathrm{fm}^{-3}$.

lations without quark degrees of freedom. The inclusion of quarks will certainly change the freezeout temperature, because the freezeout energy density is proportional to the degeneracy factor of partons, $e_{c} \sim\left(d_{G}+d_{Q}\right) T_{c}^{4}$, where $d_{G}=16$ for gluons and $d_{Q}=24$ for quarks with two flavors. For the same freezeout energy density the freezeout temperature will be a factor of 1.26 smaller when the quarks are included and if all the partons are chemically 
equilibrated. Although the initial energy is slightly enlarged when including quarks, we do not expect much stronger transverse flow at freezeout, as long as the shear viscosity of the parton system is the same with or without quarks. Therefore, the inclusion of quarks might reduce the mean $p_{T}$ by a factor of 1.26 , which then would lead to a fair agreement between the calculated and the experimentally measured mean $p_{T}$ via parton-hadron duality. If the integrated $v_{2}$ is still comparable with the experimental data, then $v_{2}\left(p_{T}\right)$ will also come to a closer agreement with the data. Work in this direction is in progress.

The parton-hadron duality picture is consistent with the hadronization in a thermalized expanding matter, which assumes that both constituents of the bulk matter at the beginning and at the end of the hadronization will be distributed according to thermal statistics. If the temperature does not change much during the phase transition, the integrated $v_{2}, v_{2}\left(p_{T}\right)$ and the $p_{T}$ spectra of pions are almost the same as those of partons. Although the energy density of pions is smaller than that of partons, the matter will expand continuously during the hadronization, which leaves the total energy, and the total particle number and, thus, the entropy of the constituents approximately unchanged. Within the picture of a thermalized expanding source the dependence of $v_{2}\left(p_{T}\right)$ on the hadron mass was also understood [6] and agrees with the RHIC data at lower $p_{T}<1.5 \mathrm{GeV}[4,5]$.

On the other hand, the STAR data [13, 35] confirmed quark recombination models [31, 32, 33, 34] for hadronization of quarks with intermediate $p_{T} \sim 1-2 \mathrm{GeV}$. These models lead to $v_{2}^{\text {hadron }}\left(p_{T}\right) \approx n v_{2}^{\text {quark }}\left(p_{T} / n\right)$ for $n=2$ for mesons and for $n=3$ for baryons, which is obviously different from the picture of a thermalized expanding source. How the transition from one to another hadronization scenario proceeds is not clear. Also, how gluons convert into quarks and antiquarks in the recombination models has to be understood and modeled.

We emphasize that how the partons hadronize is essential to obtain a complete picture of the generation of elliptic flow and to understand the transformation of the $v_{2}\left(p_{T}\right)$, the $p_{T}$ spectra, and the mean $p_{T}$ from the partonic to the hadronic phase in a consistent manner. Quantitative calculations modeling hadronization may give a more detailed understanding of this subject. As a starting point, an algorithm employing a Cooper-Frye prescription [24] will be implemented in BAMPS in the near future. Detailed comparisons with results from recent calculations using viscous hydrodynamics [36] will be given.

Finally, the gluons in the present BAMPS calculations are assumed as Boltzmann particles rather than bosons because of numerical difficulties. Bose enhancement increases the 
gluon density by a factor of $\zeta(3) \approx 1.202$ for a thermal gluon system. Moreover, the enhancement at low $p_{T}$ is considerably larger than that at large $p_{T}$. This may also have a moderate effect on the $p_{T}$ dependence of the elliptic flow and thus will be investigated in more detail.

\section{DEPENDENCE OF THE ELLIPTIC FLOW ON THE FREEZEOUT CONDI- TION}

The elliptic flow $v_{2}$ will be continuously built up if the spatial anisotropy of the particle system still exists and sufficiently strong interactions among particles are present. The buildup of $v_{2}$ does not necessarily mean that $v_{2}$ becomes always larger. After the time when the eccentricity crosses zero the system will turn spatially anisotropic again but with the sign opposite to the eccentricity of the initial one. Further evolution will then decrease $v_{2}$. Whether or not a generation of negative $v_{2}$ occurs depends on whether the kinetic freezeout of the particle system happens after or before the time when the initial eccentricity crosses zero.

In this section we discuss the dependence of the elliptic flow on the freezeout condition in $\mathrm{Au}+\mathrm{Au}$ collisions at the RHIC energy. In the present BAMPS calculations the kinetic and chemical freezeouts are assumed to occur at the same time when the local energy density drops below the chosen cutoff value of $e_{c}$. The cutoff $e_{c}$ serves as a critical energy density, at which gluons are converted to hadrons. After the phase transition no hadronic interactions take place in the present version of BAMPS. We have already speculated that a realistic modeling of hadronization may change the pattern of $v_{2}\left(p_{T}\right)$ in various ways. Moreover, a hadronic cascade simulating the decoupling stage should be included [37, 38, 39]. With the hadronic cascade, the kinetic freezeout happens later than the hadronization and the final elliptic flow will likely be different from that obtained in the present BAMPS calculations. However, this difference is expected to be rather marginal, because the viscosity in the hadron gas is much larger than that in the QGP.

Although the freezeout is considered in a simple way in the present BAMPS, it is useful to study the uncertainty in the calculated $v_{2}$, if the value of the critical energy density $e_{c}$ is varied to change the lifetime of the QGP. If the freezeout condition constrains the elliptic flow, then it also constrains the to be deduced shear viscosity of the QGP. For this purpose, 
we have performed calculations with $e_{c}=0.6 \mathrm{GeV} \mathrm{fm}^{-3}$ in addition to those with $e_{c}=1$ $\mathrm{GeV} \mathrm{fm}^{-3}$. The results are shown in Figs. $1-3$ and 5 by green curves with open triangles $\left(\alpha_{s}=0.3\right)$ and purple curves with open diamonds $\left(\alpha_{s}=0.6\right)$.

We realize that the $v_{2}$ results with $\alpha_{s}=0.3$ and $e_{c}=0.6 \mathrm{GeV} \mathrm{fm}^{-3}$ (green curves with open triangles) are almost identical to those with $\alpha_{s}=0.6$ and $e_{c}=1 \mathrm{GeV} \mathrm{fm}^{-3}$ (red curves with open squares). Stronger interactions or longer QGP phase leads to the same final values of $v_{2}$. Figure 6 shows the $v_{2}$ generation as a function of time in a $\mathrm{Au}+\mathrm{Au}$ collision with an impact parameter of $b=8.6 \mathrm{fm}$. No decrease of $v_{2}$ is observed. This indicates that the

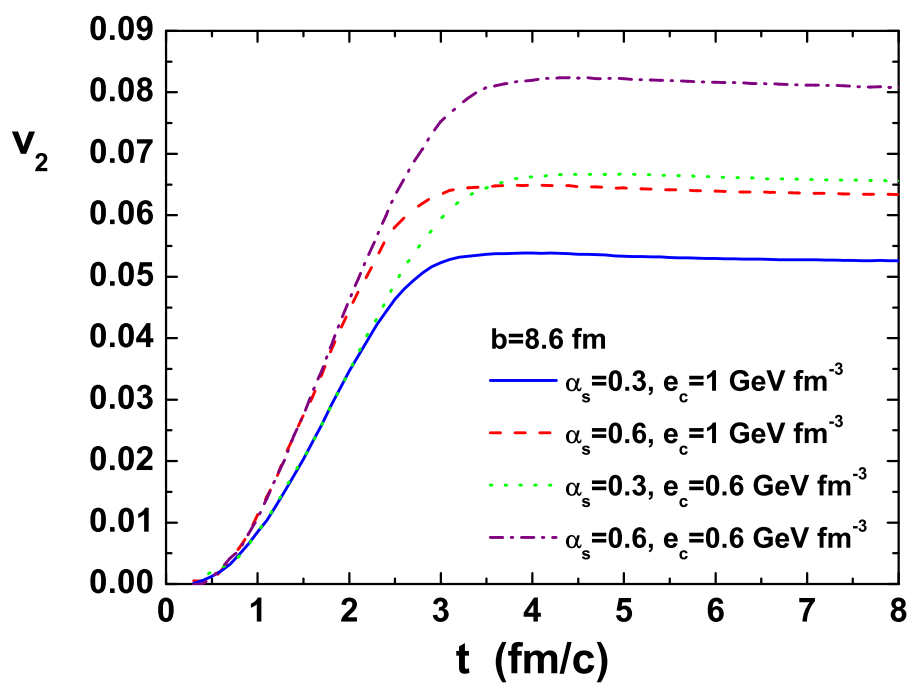

FIG. 6: (Color online) Time evolution of the elliptic flow from the BAMPS calculations in $\mathrm{Au}+\mathrm{Au}$ collisions at $\sqrt{s_{N N}}=200 \mathrm{GeV}$ at an impact parameter of $b=8.6 \mathrm{fm}$.

freezeout occurs before the initial spatial anisotropy vanishes. The saturation of $v_{2}$ begins at $2.5 \mathrm{fm} / \mathrm{c}$ for $e_{c}=1 \mathrm{GeV} \mathrm{fm}^{-3}$ and later at $3 \mathrm{fm} / \mathrm{c}$ for $e_{c}=0.6 \mathrm{GeV} \mathrm{fm}^{-3}$. The continuous increase of $v_{2}$ after $2.5 \mathrm{fm} / \mathrm{c}$ in the case for $e_{c}=0.6 \mathrm{GeV} \mathrm{fm}^{-3}$ is as strong as that before. The difference of the pressure gradient between the $x$ and $y$ directions is still large at 2.5 $\mathrm{fm} / \mathrm{c}$, whereas at this time the freezeout at $e_{c}=1 \mathrm{GeV} \mathrm{fm}^{-3}$ is nearly complete. Therefore, the uncertainty in the final elliptic flow due to the different freezeout condition is not small. In addition to the hadronization, the time scale when the hadronization occurs affects also the absolute value of $v_{2}$ as well as the transverse momentum dependence of $v_{2}$.

A detailed study of the elliptic flow is important, because this collective effect of QCD matter quantitatively constrains the shear viscosity of the medium. In Ref. [12] we have 
demonstrated within the BAMPS calculations that the shear viscosity to the entropy density ratio $\eta / s$ is nearly constant in time and approximately depends only on the coupling constant $\alpha_{s}: \eta / s \approx 0.15$ for $\alpha_{s}=0.3$ and $\eta / s \approx 0.08$ for $\alpha_{s}=0.6$. From Fig. 1 we see that with either $\alpha_{s}=0.3$ and $e_{c}=0.6 \mathrm{GeV} \mathrm{fm}^{-3}$ or $\alpha_{s}=0.6$ and $e_{c}=1 \mathrm{GeV} \mathrm{fm}^{-3}$ the same values of the final $v_{2}$ are obtained and which agree very well with the experimental data. Therefore, according to the present study, $\eta / s$ is most probably lying between 0.15 and 0.08 .

If the freezeout occurs at a lower energy density (or temperature), one would expect that the transverse momentum spectra will become softer and the final transverse energy will be smaller. From the lower panel of Fig. 5 we see that $d E_{T} / d \eta$ for $e_{c}=0.6 \mathrm{GeV} \mathrm{fm}^{-3}$ are only slightly smaller than those for $e_{c}=1 \mathrm{GeV} \mathrm{fm}^{-3}$. The differences in the transverse momentum spectra (not shown) are also tiny. The further decrease of the local energy density from 1 to $0.6 \mathrm{GeV} \mathrm{fm}^{-3}$ due to the longitudinal work done by the pressure is marginal, because at the late stage of expansion the system becomes dilute and thus the work done is small. Free streaming increases the transverse flow and thus effectively decreases the local energy density. Therefore, a lower energy density cutoff for the freezeout does not lead to a much smaller total transverse energy and mean transverse momentum, as seen in Fig. 5. On the other hand, the $v_{2}\left(p_{T}\right)$ for $\alpha_{s}=0.6$ and $e_{c}=0.6 \mathrm{GeV} \mathrm{fm}^{-3}$, shown by the purple curve with open diamonds in Fig. 2 and by purple curves in Fig. 3 , agree well with the data at the low $p_{T}<1.5 \mathrm{GeV}$, whereas the integrated $v_{2}$ (see the purple curve with open diamonds in Fig. 1) overestimate the data.

We note that within the same description for the gluon interactions the energy loss of high $p_{T}$ gluons is found to be in good agreement with the results from the GLV formalism when $\alpha_{s}=0.3$ is used [40]. Hence both jet-quenching phenomena and the buildup of elliptic flow are rather well described in BAMPS parton cascade calculations.

\section{SUMMARY}

Employing the pQCD based parton cascade BAMPS that includes pQCD bremsstrahlung and its backreaction we have calculated the elliptic flow $v_{2}$ in $\mathrm{Au}+\mathrm{Au}$ collisions at $\sqrt{s_{N N}}=$ $200 \mathrm{GeV}$. Hadronization is assumed to happen at a critical energy density, $e_{c}$. A gluon is then fragmented into a pion according to the parton-hadron duality assumption. The hadron $v_{2}$ is therefore identical to the final gluon $v_{2}$ within the present BAMPS calculations. 
We found that whereas the final gluon $v_{2}$ from the calculations with $\alpha_{s}=0.6$ and $e_{c}=$

$1 \mathrm{GeV} \mathrm{fm}{ }^{-3}$ agree well with the data, the transverse momentum dependence $v_{2}\left(p_{T}\right)$ are $20-50 \%$ lower then the data in each centrality class. The final gluon transverse momentum spectra are harder than the data and also the final gluon mean transverse momenta are 40-100\% larger than the data. However, the final gluon transverse energy per rapidity at midrapidity agrees with the data. This indicates that the hadronization process via the parton-hadron duality is not justified. A realistic hadronization that transforms a gluon to 1.5-2 pions on average would be expected for low $p_{T}$ gluons, whereas at intermediate $p_{T}$, quark recombination would be a better scenario of hadronization. In addition, the inclusion of quark degrees of freedom would reduce the final parton mean $p_{T}$ and would lead to a better agreement between the calculated and measured $v_{2}\left(p_{T}\right)$. This must be demonstrated in a new version of BAMPS calculations including the quark dynamics and employing various hadronization scenarios.

The value of the final gluon $v_{2}$ depends on the freezeout condition, i.e., $e_{c}$. The pressure gradient difference in the $x$ and $y$ directions at $e_{c}=1 \mathrm{GeV} \mathrm{fm}^{-3}$ is still large enough to further increase the elliptic flow if $e_{c}$ is changed to a smaller but still reasonable value. We observed that the final gluon $v_{2}$ and $v_{2}\left(p_{T}\right)$ are almost the same in the calculations with either $\alpha_{s}=0.6$ and $e_{c}=1 \mathrm{GeV} \mathrm{fm}^{-3}$ or $\alpha_{s}=0.3$ and $e_{c}=0.6 \mathrm{GeV} \mathrm{fm}^{-3}$. Stronger interactions or a later freezeout leads to the same elliptic flow. This outlines the uncertainty in the extraction of the shear viscosity in the QGP: The shear viscosity to the entropy density ratio will most probably be between 0.08 and 0.15 .

We furthermore conclude that adding quark degrees of freedom into the dynamical evolution of the QCD matter with a detailed understanding of the hadronization of gluons and quarks will be helpful in explaining the viscous facets of the final hadron elliptic flow and in extracting the shear viscosity to the entropy density ratio of the QGP. The results presented in this article motivate more detailed investigations of this issue in future works.

\section{Acknowledgments}

The authors thank M. Bleicher, G. Burau, and H. Stöcker for enlightening discussions. The BAMPS simulations were performed at the Center for Scientific Computing of Goethe University. This work was financially supported by the Helmholtz International Center for 
FAIR within the framework of the LOEWE program (Landes-Offensive zur Entwicklung Wissenschaftlich-ökonomischer Exzellenz) launched by the State of Hesse.

\section{APPENDIX A: NUMBER OF PARTICIPATING NUCLEONS $N_{\text {part }}$ AND CEN- TRALITY CLASSES}

The number of participating nucleons $N_{\text {part }}(b)$ in an A+B collision at an impact parameter of $b$ is calculated within the description of wounded nucleons [27]:

$$
N_{\text {part }}(b)=\int d^{2} s n_{\text {part }}(\vec{s}, \vec{b})
$$

where

$$
n_{\text {part }}(\vec{s}, \vec{b})=T_{A}(\vec{s})\left[1-e^{-\sigma_{H} T_{B}(\vec{b}-\vec{s})}\right]+T_{B}(\vec{b}-\vec{s})\left[1-e^{-\sigma_{H} T_{A}(\vec{s})}\right] .
$$

$\sigma_{H}$ denotes the nucleon-nucleon total inelastic cross section with diffraction production excluded and is set to be $\sigma_{H}=42 \mathrm{mb} . T_{A}(\vec{s})$, also $T_{B}(\vec{s})$, is the thickness function defined as

$$
T_{A}(\vec{s})=\int_{-\infty}^{\infty} d z \rho_{A}(\vec{s}, z)
$$

where $\rho_{A}(\vec{s}, z)$ is the single nucleon density and $\int d^{3} r \rho_{A}(\vec{r})=\int d^{2} s T_{A}(\vec{s})=A$. We use the Woods-Saxon function for the nucleon density

$$
\rho_{A}(\vec{r})=\rho_{A}(r)=\frac{n_{0}}{1+e^{\frac{r-R_{A}}{d}}},
$$

where $d=0.54 \mathrm{fm}, n_{0}=0.17 \mathrm{fm}^{-3}$, and $R_{A}=1.12 A^{1 / 3}-0.86 A^{-1 / 3}=6.37 \mathrm{fm}$ for $A=197$ of a $\mathrm{Au}$ nucleus.

We use $N_{\text {part }}(b)$ to make a relation between the intervals of the centrality class and the

impact parameter $b$. For an interval of $b,\left[\bar{b}_{1} ; \bar{b}_{2}\right]$, the average $N_{\text {part }}$ and the average impact parameter can be calculated by

$$
\begin{aligned}
& \left.\left\langle N_{\text {part }}\right\rangle\right|_{\left[\bar{b}_{1} ; \bar{b}_{2}\right]}=\frac{\int_{\bar{b}_{1}}^{\bar{b}_{2}} d b b N_{\text {part }}(b)}{\int_{\bar{b}_{1}}^{\bar{b}_{2}} d b b}=\frac{2}{\bar{b}_{2}^{2}-\bar{b}_{1}^{2}} \int_{\bar{b}_{1}}^{\bar{b}_{2}} d b b N_{\text {part }}(b) \\
& \left.\langle b\rangle\right|_{\left[\bar{b}_{1} ; \bar{b}_{2}\right]}=\frac{\int_{\bar{b}_{1}}^{\bar{b}_{2}} d b b^{2}}{\int_{\bar{b}_{1}}^{\bar{b}_{2}} d b b}=\frac{2}{3} \frac{\bar{b}_{2}^{3}-\bar{b}_{1}^{3}}{\bar{b}_{2}^{2}-\bar{b}_{1}^{2}} .
\end{aligned}
$$

The number of events, $N_{\text {events }}$, within $\left[\bar{b}_{1} ; \bar{b}_{2}\right]$ is proportional to $\bar{b}_{2}^{2}-\bar{b}_{1}^{2}$. Therefore,

$$
\frac{\left.N_{\text {events }}\right|_{\left[\bar{b}_{1} ; \bar{b}_{2}\right]}}{N_{\text {events }}^{M}}=\frac{\bar{b}_{2}^{2}-\bar{b}_{1}^{2}}{\operatorname{Max}\left\{\bar{b}_{j+1}^{2}-\bar{b}_{j}^{2}\right\}},
$$


where $N_{\text {events }}^{M}$ is the maximum of $N_{\text {events }}$ within all the intervals $\left[\bar{b}_{j} ; \bar{b}_{j+1}\right], j=1,2, \cdots$.

We obtain the intervals of $b$ corresponding with each centrality class by tuning $\bar{b}_{j}$ s, so that $\left.\left\langle N_{\text {part }}\right\rangle\right|_{\left[\bar{b}_{j} ; \bar{b}_{j+1}\right]},\left.\langle b\rangle\right|_{\left[\bar{b}_{j} ; \bar{b}_{j+1}\right]}$ and $\left.N_{\text {events }}\right|_{\left[\bar{b}_{j} ; \bar{b}_{j+1}\right]} / N_{\text {events }}^{M}$ from Eqs. (A5)-(A7) are comparable with the experimental data given for each centrality classes. Tables I and II show the correspondences of the intervals of $b$ with the centrality classes and the comparisons between the calculated values and the data from PHOBOS [29] and STAR [13].

TABLE I: Correspondence of the impact parameter intervals with the centrality classes by matching the PHOBOS data [29].

\begin{tabular}{l|cccccc}
\hline \hline Centrality & $0-6 \%$ & $6-15 \%$ & $15-25 \%$ & $25-35 \%$ & $35-45 \%$ & $45-50 \%$ \\
{$\left[\bar{b}_{j} ; \bar{b}_{j+1}\right](\mathrm{fm})$} & {$[0 ; 3.16]$} & {$[3.16 ; 5.23]$} & {$[5.23 ; 6.95]$} & {$[6.95 ; 8.31]$} & {$[8.31 ; 9.46]$} & {$[9.46 ; 9.98]$} \\
\hline$\left\langle N_{\text {part }}\right\rangle$ PHOBOS [29] & $344 \pm 11$ & $276 \pm 9$ & $200 \pm 8$ & $138 \pm 6$ & $93 \pm 5$ & $65 \pm 4$ \\
$\left\langle N_{\text {part }}\right\rangle$ Eq. (A5) & 350 & 282 & 208 & 147 & 101 & 74 \\
$N_{\text {events }} / N_{\text {events }}^{M}$ PHOBOS [29] & 0.505 & 0.883 & 0.997 & 0.997 & 1.0 & 0.5 \\
$N_{\text {events }} / N_{\text {events }}^{M}$ Eq. (A7) & 0.477 & 0.829 & 1.0 & 0.99 & 0.98 & 0.48 \\
\hline \hline
\end{tabular}

TABLE II: Correspondence of the impact parameter intervals with the centrality classes by matching the STAR data [13].

\begin{tabular}{l|ccccccc}
\hline \hline Centrality & $0-5 \%$ & $5-10 \%$ & $10-20 \%$ & $20-30 \%$ & $30-40 \%$ & $40-50 \%$ & $50-60 \%$ \\
{$\left[\bar{b}_{j} ; \bar{b}_{j+1}\right](\mathrm{fm})$} & {$[0 ; 3.22]$} & {$[3.22 ; 4.68]$} & {$[4.68 ; 6.59]$} & {$[6.59 ; 8]$} & {$[8 ; 9.36]$} & {$[9.36 ; 10.42]$} & {$[10.42 ; 11.07]$} \\
\hline$\left\langle N_{\text {part }}\right\rangle$ STAR [13] & $352 \pm 6$ & $298 \pm 10$ & $232 \pm 10$ & $165 \pm 12$ & $114 \pm 12$ & $75 \pm 11$ & $46 \pm 9$ \\
$\left\langle N_{\text {part }}\right\rangle$ Eq. (国5) & 349 & 293 & 226 & 160 & 108 & 69 & 46 \\
$\langle b\rangle(\mathrm{fm})$ STAR [13] & $2.3 \pm 0.2$ & $4.2 \pm 0.3$ & $5.9 \pm 0.3$ & $7.6 \pm 0.49 .0 \pm 0.5$ & $10.2 \pm 0.5$ & $11.3 \pm 0.6$ \\
$\langle b\rangle(\mathrm{fm})$ Eq. (A6) & 2.15 & 3.99 & 5.69 & 7.32 & 8.70 & 9.90 & 10.75 \\
\hline \hline
\end{tabular}

BAMPS calculations are carried out for a set of discrete impact parameters $b=2,2.8$, $3.4,4,4.5,5,5.6,6.3,7,7.8,8.6,9.6,10.4$, and $11 \mathrm{fm}$. The average of an observable $\mathcal{O}$ in one centrality class is calculated by the integral

$$
\left.\langle\mathcal{O}\rangle\right|_{\left[\bar{b}_{1} ; \bar{b}_{2}\right]}=\frac{\int_{\bar{b}_{1}}^{\bar{b}_{2}} d b b \mathcal{O}(b)}{\int_{\bar{b}_{1}}^{\bar{b}_{2}} d b b}
$$


using the trapezoid formula.

[1] J.-Y. Ollitrault, Phys. Rev. D 46, 229 (1992).

[2] S. Voloshin and Y. Zhang Z. Phys. C 70, 665 (1996).

[3] S. A. Voloshin, A. M. Poskanzer, and R. Snellings, arXiv:0809.2949 [nucl-ex].

[4] S. S. Adler et al. (PHENIX Collaboration), Phys. Rev. Lett. 91, 182301 (2003).

[5] J. Adams et al. (STAR Collaboration), Phys. Rev. Lett. 92, 052302 (2004).

[6] P. Huovinen, P. F. Kolb, U. W. Heinz, P. V. Ruuskanen, and S. A. Voloshin, Phys. Lett. B 503, 58 (2001).

[7] R. Lacey et al., Phys. Rev. Lett. 98, 092301 (2007).

[8] L. W. Chen, V. Greco, C. M. Ko, and P. F. Kolb, Phys. Lett. B 605, 95 (2005).

[9] Z. Xu and C. Greiner, Phys. Rev. C 71, 064901 (2005).

[10] Z. Xu and C. Greiner, Phys. Rev. C 76, 024911 (2007).

[11] A. El, Z. Xu, and C. Greiner, Nucl. Phys. A 806, 287 (2008).

[12] Z. Xu, C. Greiner, and H. Stöcker, Phys. Rev. Lett. 101, 082302 (2008).

[13] J. Adams et al. (STAR Collaboration), Phys. Rev. C 72, 014904 (2005).

[14] B. B. Back et al. (PHOBOS Collaboration), Phys. Rev. C 72, 051901(R) (2005).

[15] Z. Xu and C. Greiner, Phys. Rev. Lett. 100, 172301 (2008).

[16] P. Romatschke and U. Romatschke, Phys. Rev. Lett. 99, 172301 (2007).

[17] T. S. Biro, E. van Doorn, B. Müller, M. H. Thoma, and X.-N. Wang, Phys. Rev. C 48, 1275 (1993).

[18] S. M. H. Wong, Nucl. Phys. A 607, 442 (1996).

[19] J. F. Gunion and G. Bertsch, Phys. Rev. D 25, 746 (1982).

[20] P. Danielewicz and G. F. Bertsch, Nucl. Phys. A 533, 712 (1991).

[21] A. Lang, H. Babovsky, W. Cassing, U. Mosel, H. G. Reusch, and K. Weber, J. Comp. Phys. 106, 391 (1993).

[22] W. Cassing, Nucl. Phys. A 700, 618 (2002).

[23] G. Ferini, M. Colonna, M. Di Toro, and V. Greco, arXiv:0805.4814[nucl-th].

[24] H. Petersen, J. Steinheimer, G. Burau, M. Bleicher, and H. Stöcker, Phys. Rev. C 78, 044901 (2008). 
[25] S. A. Bass et al., Prog. Part. Nucl. Phys. 41, 255 (1998); M. Bleicher et al., J. Phys. G 25, 1859 (1999).

[26] K. J. Eskola, K. Kajantie, and J. Lindfors, Nucl. Phys. B 323, 37 (1989).

[27] A. Bialas, M. Bleszynski and W. Czyz, Nucl. Phys. B 111, 461 (1976); Jean-Paul Blaizot, Phuong Mai Dinh, and Jean-Yves Ollitrault, Phys. Rev. Lett. 85, 4012 (2000).

[28] A. Adare et al. (PHENIX Collaboration), Phys. Rev. Lett. 98, 162301 (2007).

[29] B. B. Back et al. (PHOBOS Collaboration), Phys. Lett. B 578, 297 (2004).

[30] J. Adams et al. (STAR Collaboration), Phys. Rev. C 70, 054907 (2004).

[31] Z. W. Lin and C. M. Ko, Phys. Rev. Lett. 89, 202302 (2002); V. Greco, C. M. Ko, and P. Levai, Phys. Rev. Lett. 90, 202302 (2003).

[32] S. Voloshin, Nucl. Phys. A 715, 379c (2003); D. Molnar and S. A. Voloshin, Phys. Rev. Lett. 91, 092301 (2003).

[33] R. J. Fries, B. Müller, C. Nonaka, and S. A. Bass, Phys. Rev. Lett. 90, 202303 (2003).

[34] R. C. Hwa and C. B. Yang, Phys. Rev. C 67, 064902 (2003).

[35] J. Adams et al. (STAR Collaboration), Phys. Rev. Lett. 92, 052302 (2004).

[36] K. Dusling and D. Teaney, Phys. Rev. C 77, 034905 (2008); H. Song and U. W. Heinz, Phys. Rev. C 77, 064901 (2008); M. Luzum and P. Romatschke, Phys. Rev. C 78, 034915 (2008).

[37] X. Zhu, M. Bleicher, and H. Stöcker, Phys. Rev. C 72, 064911 (2005).

[38] T. Hirano, U. W. Heinz, D. Kharzeev, R. Lacey, and Y. Nara, Phys. Lett. B 636, 299 (2006).

[39] J. Bleibel, G. Burau, A. Faessler, and C. Fuchs, Phys. Rev. C 76, 024912 (2007).

[40] O. Fochler, Z. Xu, and C. Greiner, arXiv:0806.1169[hep-ph]. 